\title{
Testing the Cactus code on exact solutions of the Einstein field equations
}

\author{
Dumitru N. Vulcanov* and Miguel Alcubierre ${ }^{\dagger}$ \\ Max-Planck-Institut für Gravitationsphysik \\ Albert-Einstein-Institut \\ Numerical Relativity Group \\ Golm, Am Mühlenberg 1, D-14476, Germany
}

\begin{abstract}
The article presents a series of numerical simulations of exact solutions of the Einstein equations performed using the Cactus code, a complete 3-dimensional machinery for numerical relativity. We describe an application ("thorn") for the Cactus code that can be used for evolving a variety of exact solutions, with and without matter, including solutions used in modern cosmology for modeling the early stages of the universe. Our main purpose has been to test the Cactus code on these wellknown examples, focusing mainly on the stability and convergence of the code.
\end{abstract}

\section{Introduction}

Numerical Relativity is concerned with the study of numerical solutions of the Einstein's equations for the gravitational field, which are at the core of the theory of General Relativity. General Relativity ([1]-[2]) is a 4-dimensional theory involving one dimension of time and three of space. The field equations, called Einstein equations, are :

$$
R_{i j}-\frac{1}{2} g_{i j} R+\lambda g_{i j}=\frac{8 \pi G}{c^{4}} T_{i j}
$$

*Permanent address : The West University of Timişoara, Theoretical and Computational Physics Department, B-dul V. Pârvan no. 4, 1900 Timişoara, România, e-mail : vulcan@physics.uvt.ro

${ }^{\dagger}$ e-mail : miguel@aei.mpg.de 
where $\lambda$ is the cosmological constant, $R_{i j}$ the Ricci tensor, $R$ the Ricci scalar, $g_{i j}$ the spacetime metric, $T_{i j}$ the stress-energy tensor, $\mathrm{G}$ the gravitational constant, $c$ the speed of light and $i, j=0,1,2,3$.

These equations are an extremely complicated system of coupled, non-linear, partial differential equations and solving them numerically makes enormous demands on the processing power and memory of a computer. Because of this, Numerical Relativity (沺, [5]) has proceeded in several stages, first solving 1-dimensional problems (that is 1 spatial dimension, e.g. spherical symmetry), and then moving to 2-dimensional problems (i.e. axial symmetry). Only in the last few years have computers developed sufficiently to consider tackling fully 3-dimensional problems. At the Albert Einstein Institute, an international team led by Edward Seidel has developed a complete 3D code for numerical relativity, which has been named the "Cactus code" (see [6]). The Cactus code has been mainly designed as a computational toolkit (freely available for the scientific community) for simulating different systems of partial differential equations. In the particular of relativity, the code can be used to simulate systems with strong gravitational fields: collapsing gravitational waves, colliding black-holes, neutron stars, and other violent astrophysical processes generating gravitational waves [7]-13].

Among the main problems related to the development of the Cactus code has been that of testing the code for stability and convergence during the simulations. Several applications of the code (named generically "thorns") were designed for this purpose, one of them being the so-called thorn "Exact", were some known exact solutions of the vacuum Einstein equations were implemented. The original version of this thorn was written and has been furthered developed by many people. Thorn Exact was designed for a comparative study of the numerical evolution of exact solutions of the vacuum Einstein equations [7]. The thorn requires the full 4-dimensional metric of a given exact solution in the whole spacetime. Its routines then generate $3+1$ data (i.e. lapse function, shift vector, spatial metric, and extrinsic curvature) from the given exact solution both as initial data for the numerical evolution, and also at every iteration step of the evolution so that it can be directly compared with the numerically evolved data. A limited number of exact solutions were included (among them Minkowski, Novikov and several Black Hole spacetimes), but since the thorn was constructed in very modular form it has been easy to add new exact solutions by just writing a special subroutine for each new case (with the corresponding parameters). Thus we have added a series of space-times which are of cosmological interest, such as De Sitter, Friedmann, Kasner, DeMilne and Gödel models. The main problem was that some of these models are exact solutions of the Einstein equations with matter, so the original thorn has to be generalized to allow one to introduce the components of the corresponding stress-energy tensor. 
The current version of this thorn Exact now contains also several space-time models used in cosmology for treating the early stages of the universe. Thus, through this thorn, the Cactus code can be used for numerical cosmology studies, an example being the inflationary cosmology where inflation is controlled by a scalar field (this is the main purpose of our future work).

This article is a report on the current status of thorn Exact. We shall present some of the numerical results obtained on running the Cactus code for several cosmological models introduced in this thorn. We point out here also how the code produces convergent numerical results and how stable the evolution can be for different cases. At the moment the Cactus code can use two different formulations for numerical integrating the Einstein equations, namely the standard Arnowitt-Deser-Misner formulation ([1], [3]) (implemented as the ADM thorn) and a recent reformulation introduced by Baumgarte and Shapiro ([1], [14]) based on previous work of Shibata and Nakamura ([15]) (implemented in the BSSN thorn). The comparative study of these two formulations shows the better stability properties of the BSSN formulation (as already pointed out in [11]). A special point to mention here is the problem of the boundary behavior of the numerical simulations. The boundary conditions currently available in the Cactus code are specifically adapted for the simulations of either asymptotically flat spacetimes, or periodic spacetimes. Because of this we have been forced to use special boundary conditions in thorn Exact which force the code to take the boundary values from the exact solution itself, except in those cases were we can use the "flat" boundary condition already implemented in the code (the flat boundary condition means that the value of the given field or fields at the boundary is simply copied from the value one grid point in along the direction normal to the boundary).

In the next sections we shall present several results obtained after running Cactus for different metrics given by thorn Exact, on different computer architectures. The simulations were performed mainly on single processor machines, using both the AEI computer network (SGI or Dec machines with UNIX operating system) and a Pentium III machine with a UNIX FreeBSD operating system at the West University of Timişoara. Some of the simulations, with similar results, were also done on the Origin 2000 supercomputer at the AEI. As a conclusion of our work we have extended the Exact thorn to deal with exact solutions of the Einstein equations with matter, in particular several cosmological solutions. The thorn is available, via internet downloading, for anyone interested in using it.

Through this article and in the Cactus code we shall use geometrical units with $G=$ $c=1$. 


\section{De Sitter cosmologies}

Modern cosmology is based on the Robertson-Walker metric ([16], 17]) having the line element as :

$$
d s^{2}=-d t^{2}+R(t)^{2}\left[\frac{d r^{2}}{1-k r^{2}}+r^{2}\left(d \theta^{2}+\sin ^{2} \theta d \phi^{2}\right)\right]
$$

in spherical coordinates $(t, r, \theta, \phi)$. Here $R(t)$, the scale factor, is depending only on the time $t$. The parameter $k$ has three values, namely $k=0,1,-1$ for flat, closed or respectively open spatial three geometry. The Einstein equations (1) for $\lambda=0$ and a stress-energy tensor as for a perfect fluid

$$
T_{i j}=(p+\rho) u_{i} u_{j}+p g_{i j}
$$

(where $p$ is the pressure, $\rho$ the density and $u_{i}$ the velocity in comoving coordinates) have, as an exact solution, for a matter dominated universe $(p=0)$ and flat spatial geometry $(k=0)$ the DeSitter metric (also called Einstein-DeSitter metric, see [1], 20, [16] and [17]) where $R(t) \sim t^{2 / 3}$. Thus DeSitter metric has the line element as :

$$
d s^{2}=-d t^{2}+a t^{4 / 3}\left[d r^{2}+r^{2}\left(d \theta^{2}+\sin (\theta)^{2} d \phi^{2}\right)\right] .
$$

where $a$ is a constant. Here the only non-vanishing component of the the stress-energy tensor is :

$$
T_{00}=\frac{1}{6 \pi t^{2}}
$$

Next we shall present some of the results obtained after running Cactus for the De Sitter metric. First we show the behavior of the radial metric function $\left(g_{r r}=R(t)^{1} /(1-\right.$ $k r^{2}$ ) in the above line element, being denoted with grr at the Cactus output) obtained after 20 iterations on a grid of $28^{3}$ points, with $0 \leq x, y, z \leq 1$ and $\Delta t=0.25 \Delta x$ (Fig. 11). Here and in the next figures, "normalized" means the L2 norm of the respective function calculated using all the values of the function on the computational grid at one time and the respective output Cactus files are denoted with _nm2 extension.

Cactus code is evolving the Einstein equations using the $3+1$ decomposition of spacetime (both in ADM or BSSN evolution methods). Thus the Einstein equations can be split in two groups : the dynamic equations (for the time derivatives of the three-dimensional metric and extrinsic curvature) and the constraint equations (the Hamiltonian constraint and the Momentum constraint) - see [1] and [3]. The constraint equations are satisfied during all time evolution of the system. Thus one of the main tests on the convergence in 

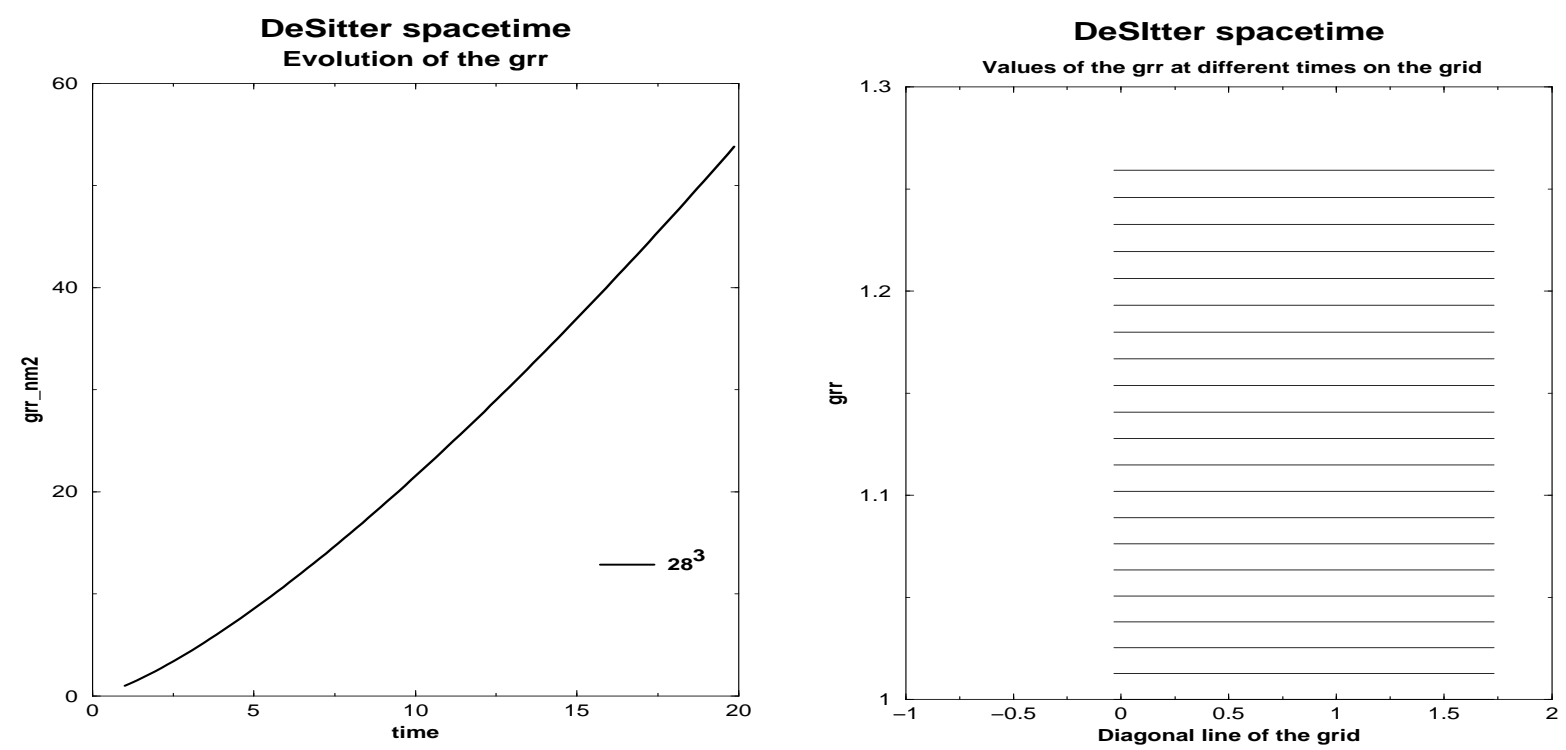

Figure 1: Evolution of the L2 norm of the radial metric function $g_{r r}$ versus time (left panel) and of the metric function itself through the grid at different times (right panel) for the De Sitter spacetime. The numerical grid has $28^{3}$ points. 

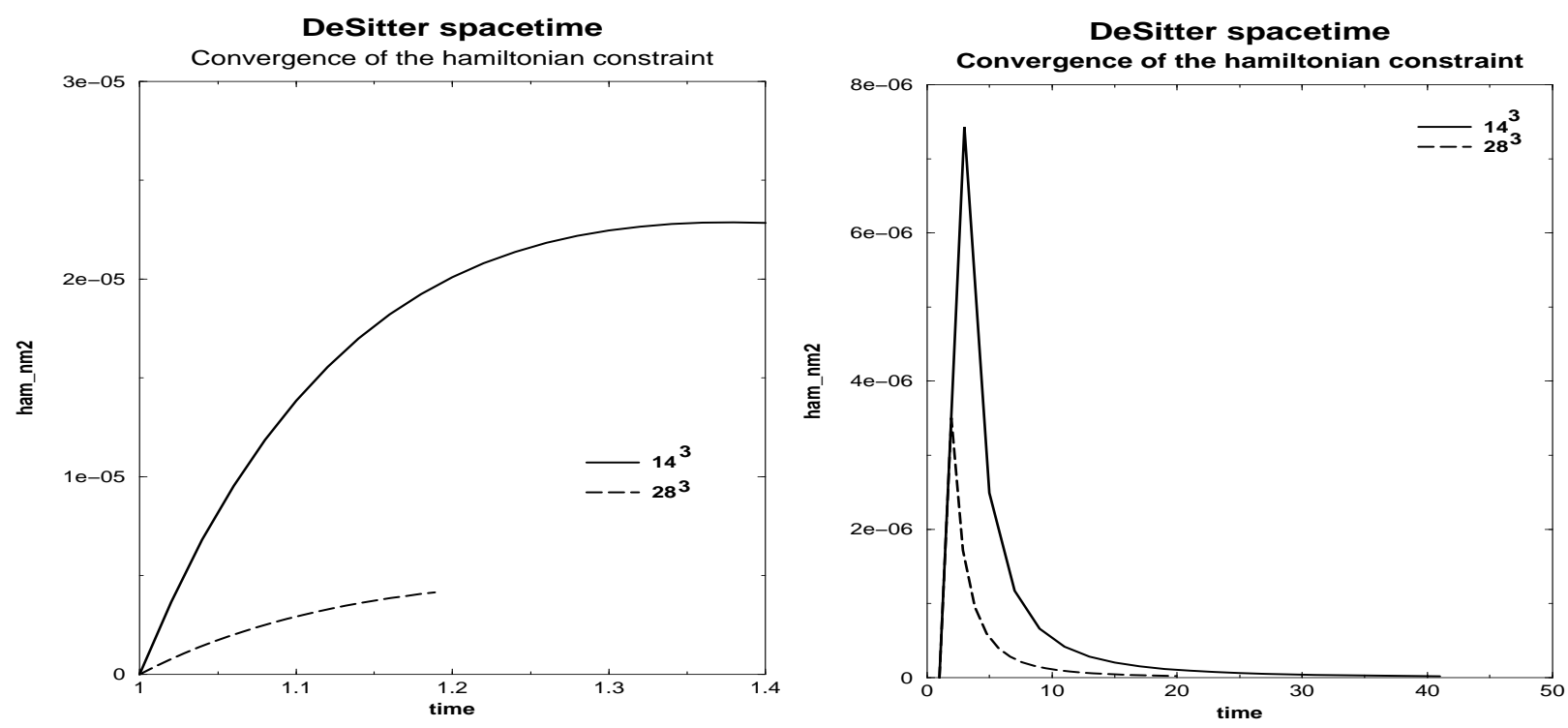

Figure 2: Convergence of the L2 norm of the Hamiltonian constraint for the De Sitter spacetime using two resolutions, one with $14^{3}$ and the second with $28^{3}$ points on the grid, for 20 iterations (left panel) and after 2000 iterations (right panel).

the Cactus code is given by the time behavior of the Hamiltonian constraint (an output of the Cactus code through a thorn called ADMConstraints, namely ham). In the next Figure 2 we show the convergence of the L2 norm of the Hamiltonian constraint for DeSitter metric, for 20 and 2000 iterations, using two different resolutions on the grid, one with $14^{3}$ and the second with $28^{3}$ points (both grids cover the same region of spacetime, so the grid with more points has a smaller value of the finite difference interval $\Delta x$ ). Notice that the Hamiltonian constraint for a true solution of the Einstein equations should be equal to zero. Finite differencing errors imply that the numerical solution will have a non-vanishing value of the Hamiltonian constraint. For a consistent finite difference approximation of the Einstein equations, we should expect the Hamiltonian constraint to approach zero as the resolution is increased. For a second order approximation, the value of the Hamiltonian constraint should go down by a factor of four when the resolution is doubled. The figure shows that we have close to second order convergence.

This was the method to test the convergence of the code in all examples presented in this article. We have obtained good second order convergence in all examples as is shown

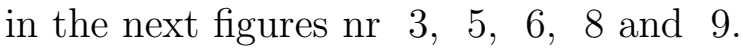



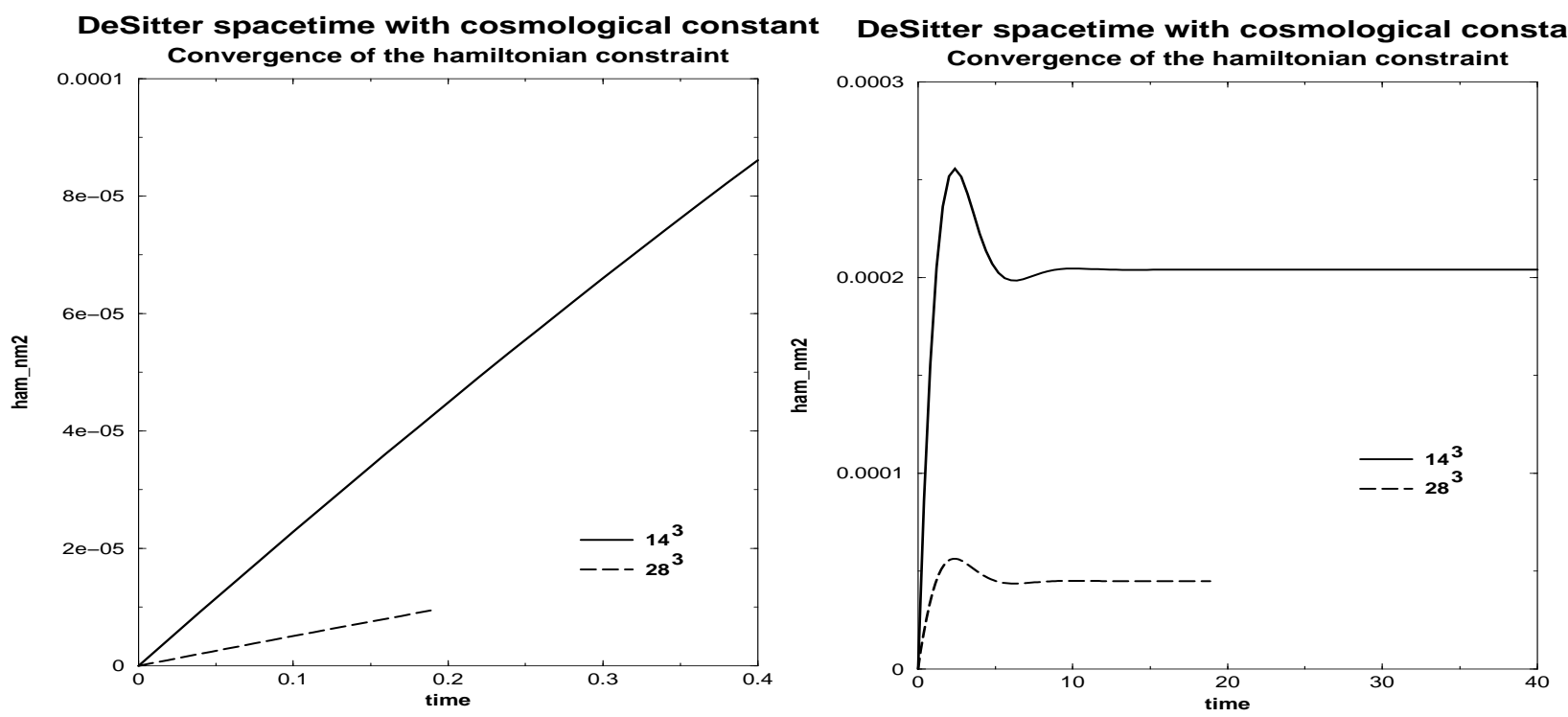

Figure 3: Convergence of the Hamiltonian constraint for the De Sitter spacetime with cosmological constant using two resolutions, one with $14^{3}$ and the second with $28^{3}$ points on the grid, for 20 iterations (left panel) and after 2000 iterations (right panel).

These results were obtained using the BSSN formulation for the numerical evolution, but we have obtained similar results using the ADM formulation. As a major conclusion we can point out that we found that the BSSN formulation results in stable evolutions. For long time evolutions (that is for more iterations than 20, for example 2000 iterations in our simulations), the Hamiltonian constraint grows from zero to a constant value, as it is obvious by inspecting all our next graphs on the convergence of the Hamiltonian constraint (see below). In our evolutions, one can also observe how the computational errors propagate back into the computational grid after being reflected on the boundary due to the use of inappropriate boundary conditions (here we used the "flat" boundary conditions implemented in the Cactus code). This will be an important problem to solve in the future. The De Sitter parameter used in these simulations was $a=1.0$ and the chosen slicing condition was an "exact" slicing (i.e. use the exact lapse coming from Exact thorn).

Another example of De Sitter cosmology is that of the De Sitter metric with cosmological constant, having the form :

$$
d s^{2}=-d t^{2}+e^{2 / 3 \sqrt{3 \lambda} t}\left(d x^{2}+d y^{2}+d z^{2}\right) .
$$


where $\lambda$ is the cosmological constant. This is an example of an exact solution of the Einstein equations (11) with cosmological constant and without matter. Here we can still use the Cactus code, even though it has been written for the case of no cosmological constant, by using a simple trick: we have transferred the term with the cosmological constant to the right hand side of the Einstein equations. Thus we shall have a nonvanishing "matter" term having as components

$$
T_{i j}=-\frac{\lambda}{8 \pi} g_{i j}=\left(\begin{array}{cccc}
\frac{1}{8} \frac{\lambda}{\pi} & 0 & 0 & 0 \\
0 & -\frac{1}{8} \frac{\lambda e^{2 / 3 \sqrt{3 \lambda} t}}{\pi} & 0 & 0 \\
0 & 0 & -\frac{1}{8} \frac{\lambda e^{2 / 3 \sqrt{3 \lambda t}}}{\pi} & 0 \\
0 & 0 & 0 & -\frac{1}{8} \frac{\lambda e^{2 / 3 \sqrt{3 \lambda} t}}{\pi}
\end{array}\right) .
$$

(remember that we have $G=c=1$ ). Introducing this in the Cactus code and taking $\lambda=1$, we have obtained the numerical results presented in the next figures 3 and 4 . First, we investigated the convergence of the code, using again the time behavior of the Hamiltonian constraint. Figure 3 shows this for simulations with 20 and 2000 iterations, using again two resolutions, one with $14^{3}$ and the second with $28^{3}$ points on the grid, with $0 \leq x, y, z \leq 6$ and $\Delta t=0.25$. We can point out the same conclusions on the convergence and the stability of the code as in the case of the De Sitter spacetime.

Figure 1 presents the evolution in time of the radial metric component $g_{r r}$ for 2000 iterations. It is easy to see here the rapid increase of the radius of the universe (which is related to $g_{r r}$ ), showing the inflationary nature of a universe modeled by this metric.

\section{Kasner solutions}

We have used the Cactus code for several cosmological solutions of the Einstein equations, generically named "Kasner" metrics, included in the class of homogeneous anisotropic models, which can emulate some early stages of the Universe. One of these metrics is the so-called "Kasner-like" metric (See [17] and [19]), which in Cartesian coordinates has the form:

$$
d s^{2}=-d t^{2}+t^{2 q}\left(d x^{2}+d y^{2}\right)+t^{2-4 q} d z^{2} .
$$



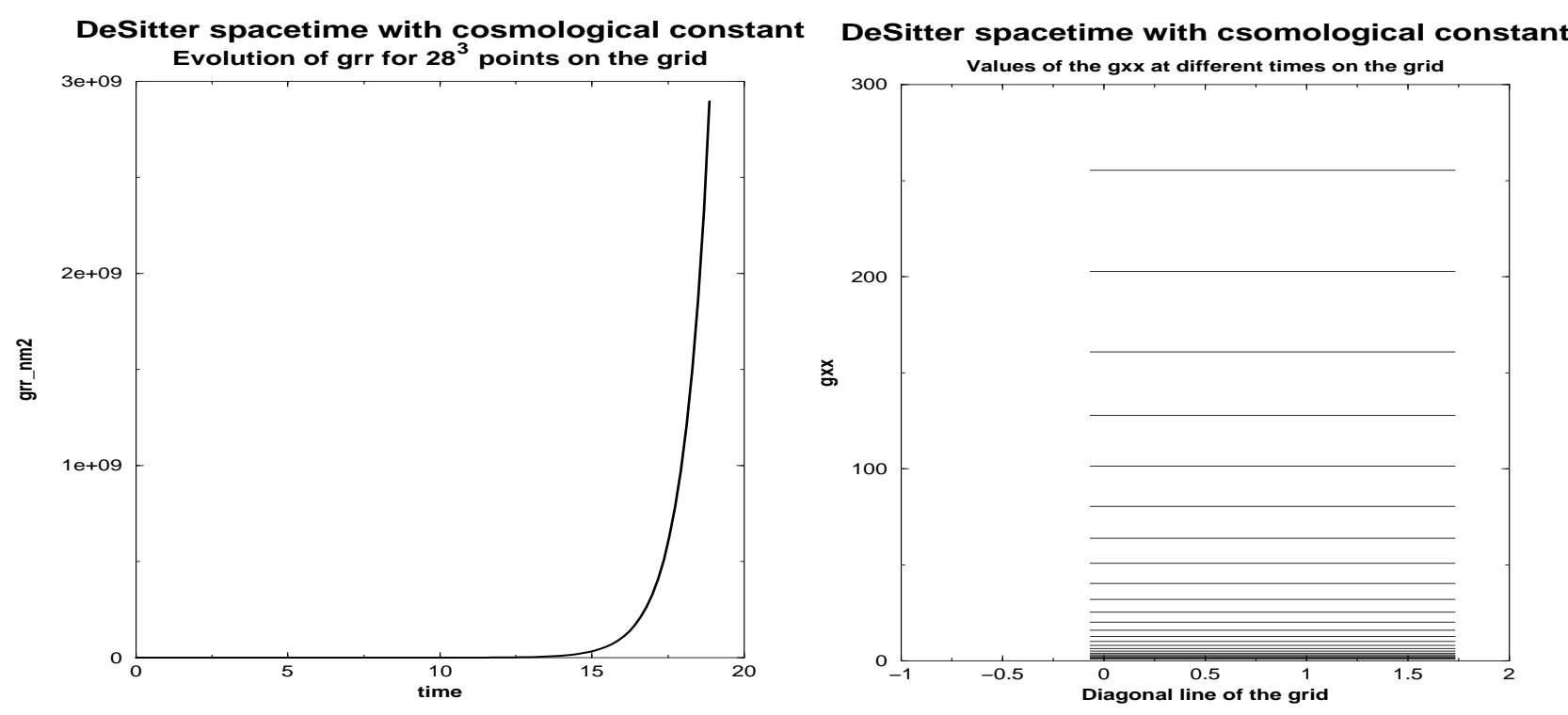

Figure 4: Behavior of the L2 norm of the radial metric component $g_{r r}$ (left panel) and its evolution in time (right panel) showing the inflationary nature of a universe modeled with the De Sitter metric with cosmological constant. 
Here we have a stress-energy tensor which has all off-diagonal components vanishing:

$$
T_{i j}=\left(\begin{array}{cccc}
q \frac{(2-3 q)}{8 \pi t^{2}} & 0 & 0 & 0 \\
0 & q \frac{(2-3 q) t^{2 q}}{8 \pi t^{2}} & 0 & 0 \\
0 & 0 & q \frac{(2-3 q) t^{2 q}}{8 \pi t t^{2}} & 0 \\
0 & 0 & 0 & q \frac{(2-3 q) t^{2-4 q}}{8 \pi t^{2}}
\end{array}\right) .
$$

This metric forms a one parameter family of solutions of Einstein's equations with a perfect stiff fluid. The parameter $q$ is related to the energy density, as is obvious from the last equation. The qualitative features of the expansion depend on $q$ in the following way: for $q>1 / 2$ the universe expands from a "cigar" singularity; for $q=1 / 2$, the universe expands purely transversally from an initial "barrel" singularity; for $0<q<1 / 2$ the initial singularity is "point-like" and if $q \leq 0$ we have a "pancake" singularity. The case $q=1 / 3$ corresponds to an isotropic universe with a stiff fluid; the case $q=0$ is a region of Minkowski spacetime in non-Cartesian coordinates. This family of metrics is "Kasnerlike" in the sense that the sum of the exponents is equal to one, but the sum of the squares is not equal to one except in the cases when $q=0$ or $q=2 / 3$, when we have the vacuum case.

We have investigated the numerical behavior of this metric for different values of the parameter $q$, looking specially at the convergence of the Hamiltonian constraint, starting from an initial time $t=1$ (since the metric is singular at $t=0$ ), using "flat" boundary conditions and, as in the previous cases, for both 20 and 2000 iterations using the BSSN formulation. All convergence tests were done with two resolutions, one with $14^{3}$ and the second with $28^{3}$ points on the grid. Figure 5 displays our results for the vacuum case $(q=2 / 3)$ and 20 iterations. As can be clearly seen the convergence of the Hamiltonian constraint is of second order. In the figure we show also the evolution of the L2 norm of the $g_{x x}$ metric component during that time. We show the same plots in figure 6, but this time for the value $q=-1$ of the Kasner parameter.

Figure 7 shows the evolution of the radial metric component for the two previous cases, pointing out some differences due to the special symmetry of this metric (see above and compare with $g_{x x}$ from the previous figures).

Next we have considered another Kasner type metric, namely the Kasner axisymmetric spacetime $([18])$ :

$$
d s^{2}=-\frac{d t^{2}}{\sqrt{t}}+\frac{d x^{2}}{\sqrt{t}}+t d y^{2}+t d z^{2} .
$$

This is an exact solution of the vacuum Einstein equations, explicitly homogeneous, and features a cosmological singularity at $t=0$. Figure 8 shows some of our results for a "long 

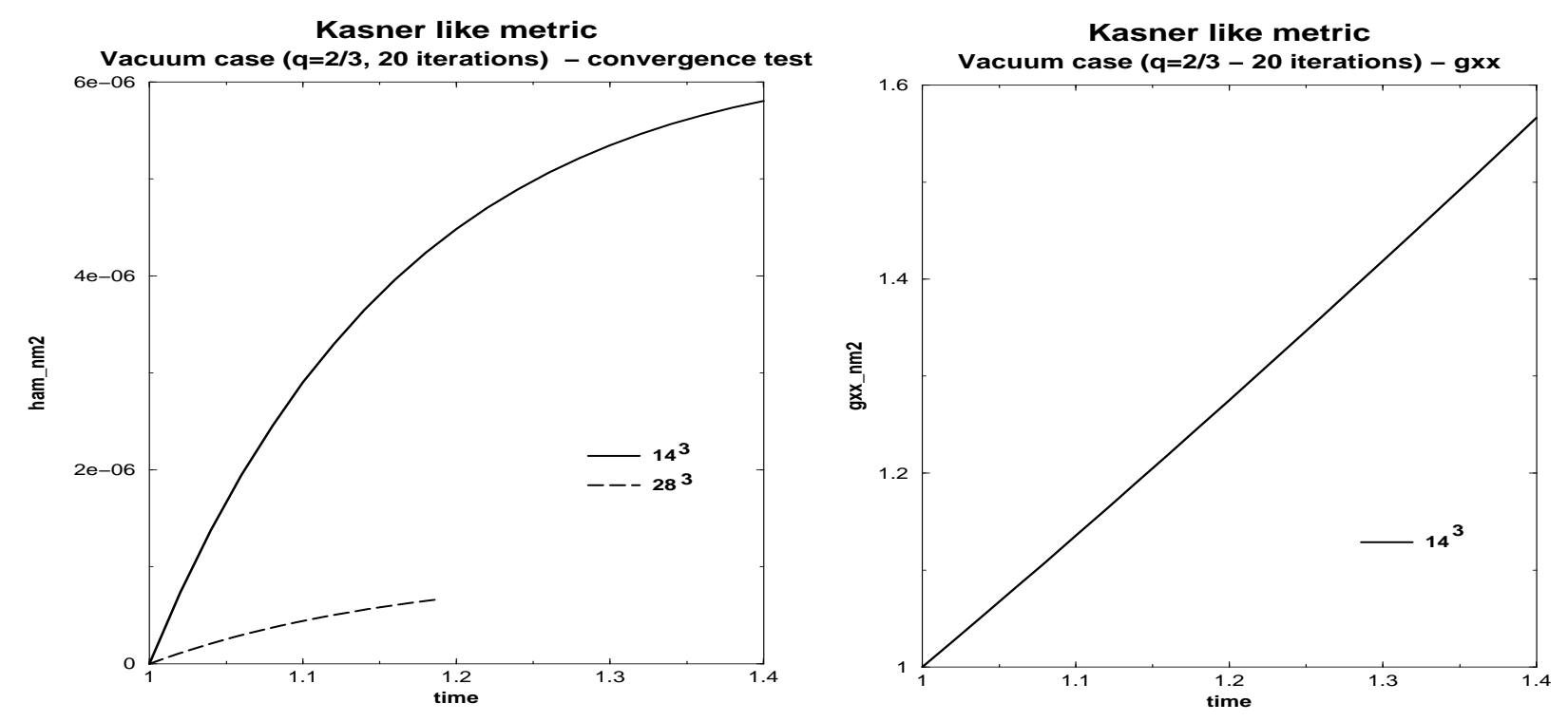

Figure 5: Convergence of the Hamiltonian constraint (left panel) and evolution of the L2 norm of the $g_{x x}$ metric component (right panel) for the Kasner-like spacetime - vacuum case $(q=2 / 3)$ and 20 iterations. 

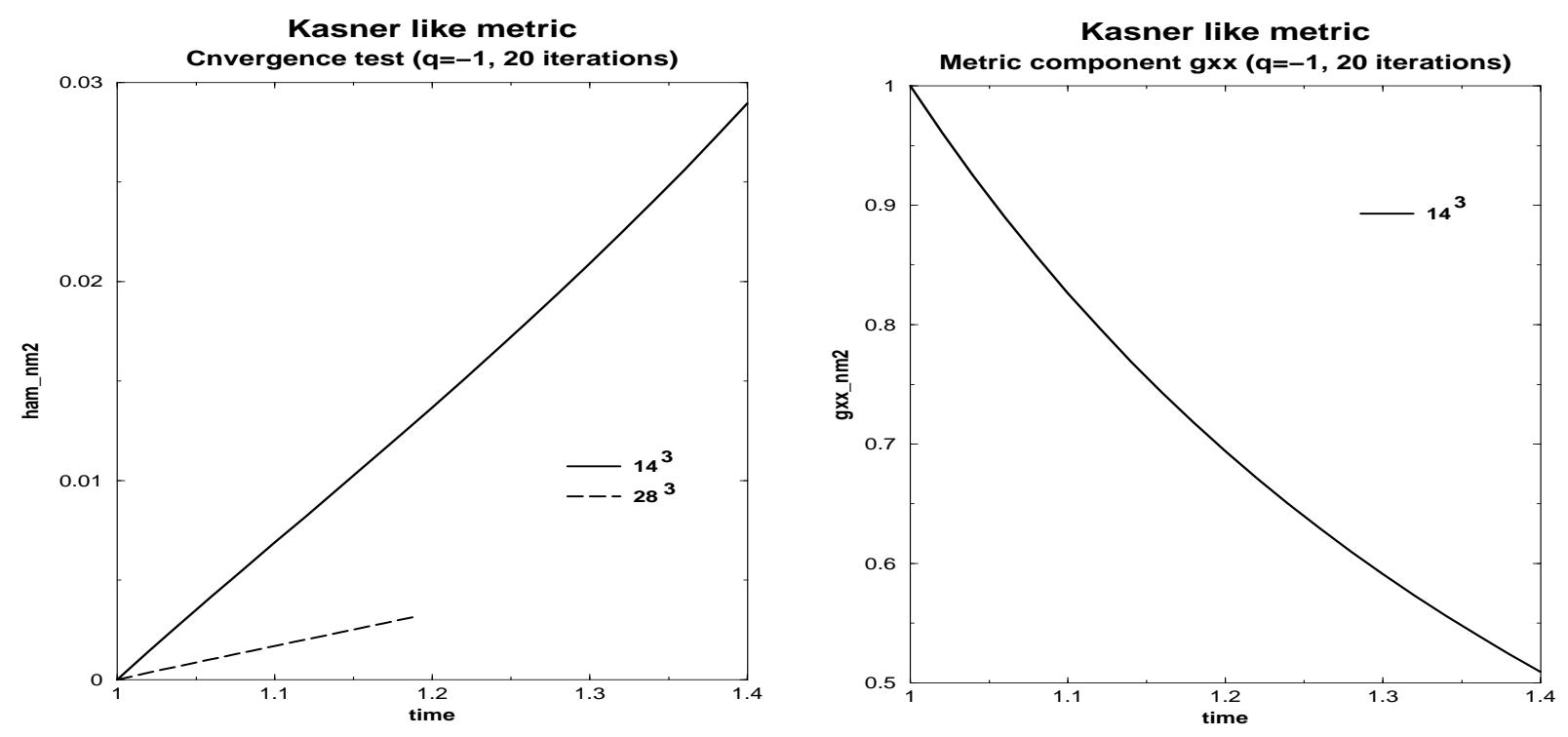

Figure 6: Convergence of the Hamiltonian constraint (left panel) and evolution of the L2 norm of the $g_{x x}$ metric component (right panel) for the Kasner-like spacetime for $q=-1$ and 20 iterations. 

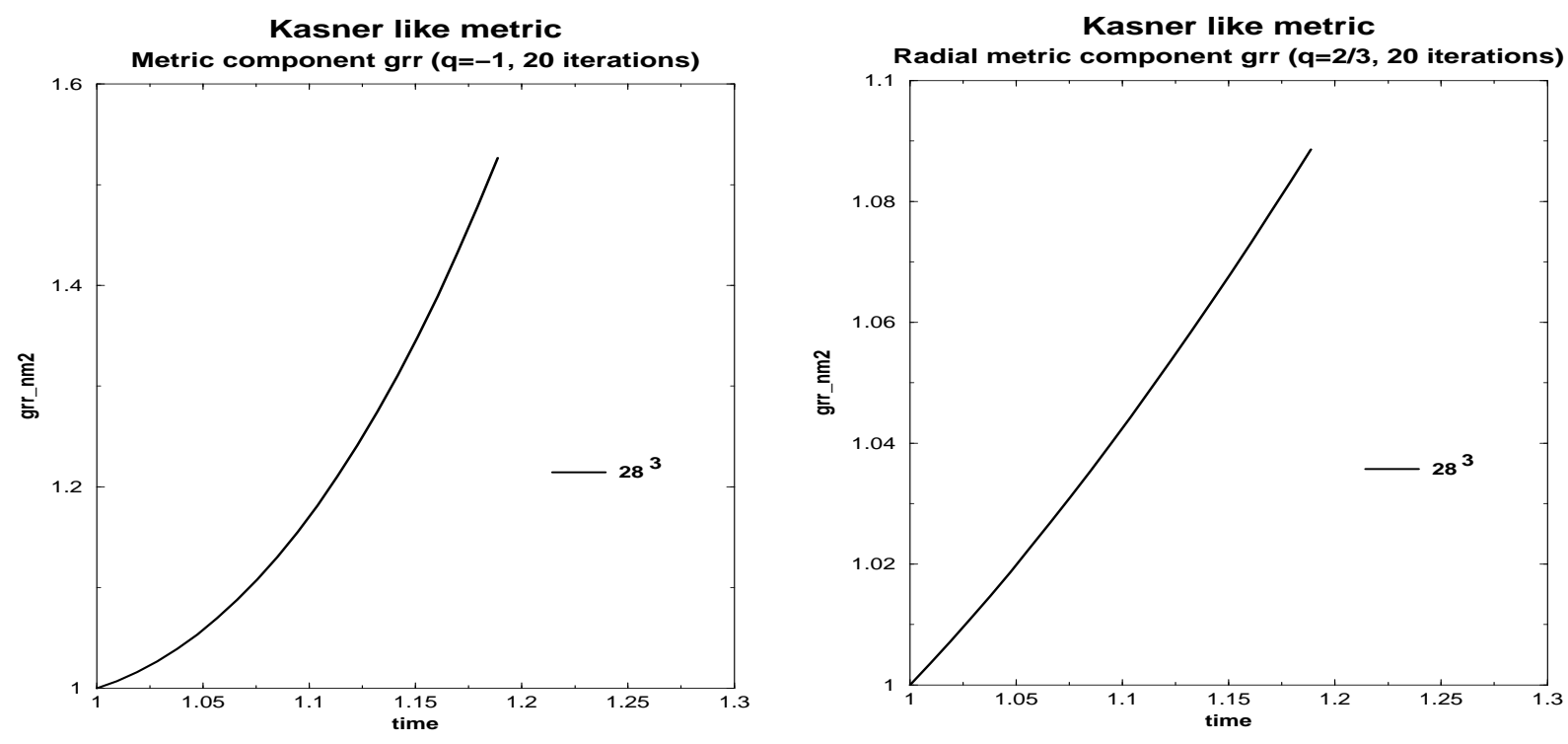

Figure 7: Evolution of the L2 norm of the radial metric component $g_{r r}$ for the vacuum case $(q=2 / 3$, right panel) and for $q=-1$ case (left panel) for the Kasner-like spacetime, with 20 iterations in both cases. 

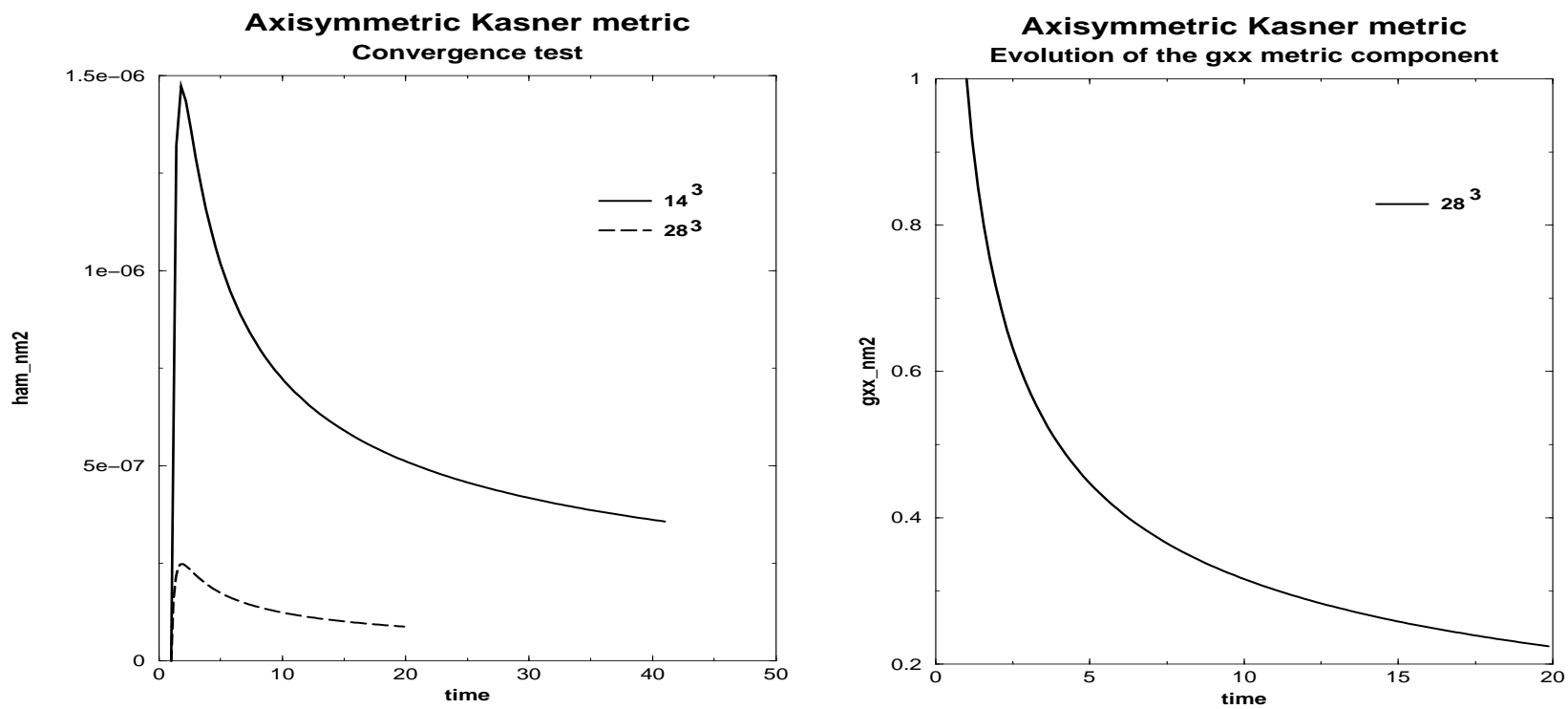

Figure 8: Convergence of the Hamiltonian constraint (left panel) and the evolution of the L2 norm of $g_{x x}$ (right panel) for the axisymmetric Kasner spacetime using 2000 iterations.

time" simulation (with 2000 iteration) showing the stability and second order convergence of the code.

A generalization of the Kasner metrics can be introduced [1] with a metric of the form:

$$
d s^{2}=-d t^{2}+t^{2 p_{1}} d x^{2}+t^{2 p_{2}} d y^{2}+t^{2 p_{3}} d z^{2},
$$

where the Kasner parameters $p_{1}, p_{2}$ and $p_{3}$ satisfy two relations:

$$
p_{1}+p_{2}+p_{3}=1 \quad \text { and } \quad p_{1}^{2}+p_{2}^{2}+p_{3}^{2}=1 .
$$

Restricting ourselves only to two parameters, $p_{1}$ and $p_{2}$, we have the following stressenergy tensor:

$$
T_{i j}=\left(\begin{array}{cccc}
\frac{A}{8 \pi t^{2}} & 0 & 0 & 0 \\
0 & \frac{A t^{2 p_{1}-2}}{8 \pi} & 0 & 0 \\
0 & 0 & \frac{A t^{2 p_{2}-2}}{8 \pi} & 0 \\
0 & 0 & 0 & \frac{A t^{-2 p_{1}-2 p_{2}}}{8 \pi}
\end{array}\right) \text {, }
$$

where $A=p_{1}-p_{1}^{2}+p_{2}-p_{2}^{2}-p_{1} p_{2}$ (note the use of the above first condition on the parameters, thus we have $\left.p_{3}=1-p_{1}-p_{2}\right)$. We have done several simulations for this metric. Figure 9 shows results for 2000 time iterations, using $p_{1}=-1 / 3, p_{2}=2 / 3$. 

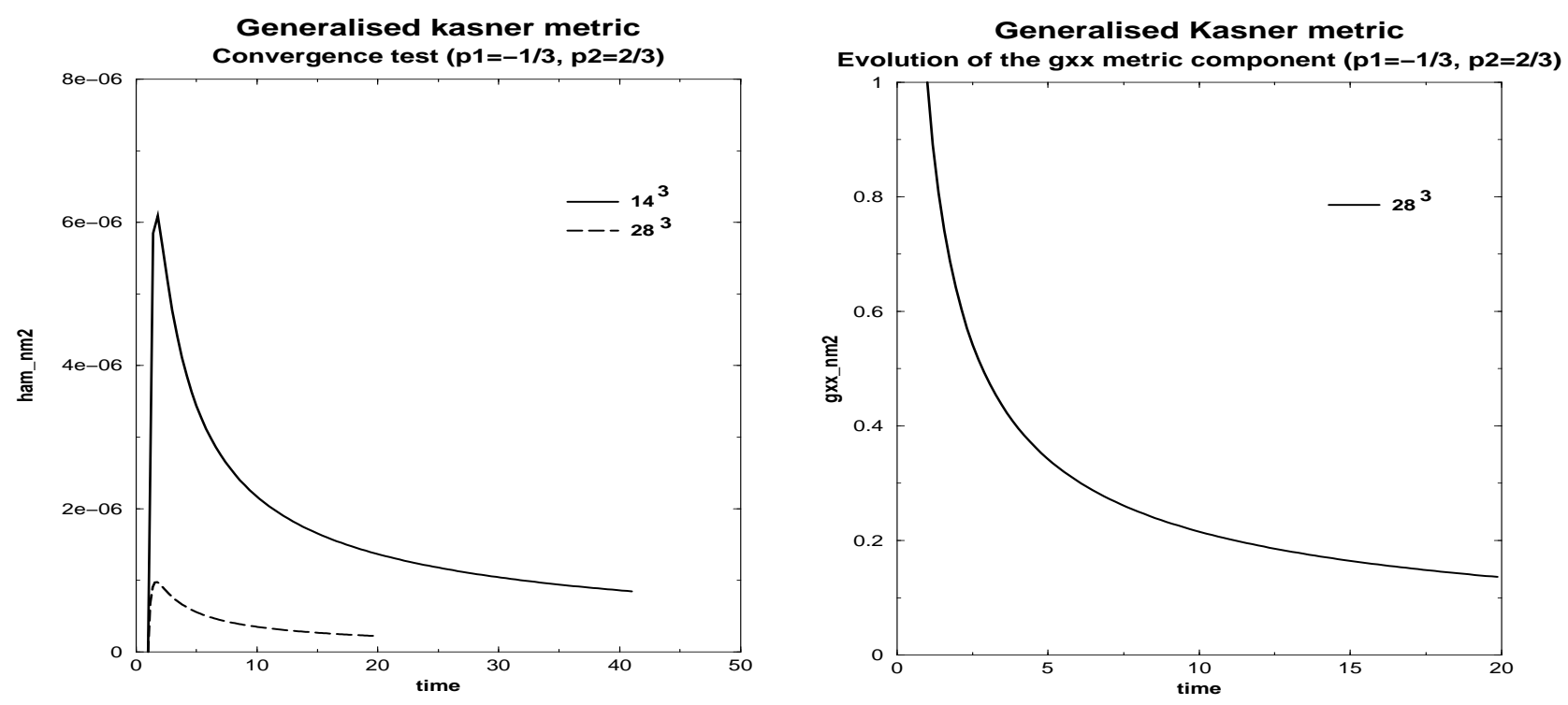

Figure 9: Convergence of the Hamiltonian constraint (left panel) and evolution of the L2 norm of $g_{x x}$ (right panel) for the generalized Kasner spacetime - $p_{1}=-1 / 3, p_{2}=2 / 3$, and 2000 iterations. 


\section{Robertson-Walker metric and other examples}

We have studied also other metrics, new or old ones form the Exact thorn. We only mention here the Bianchi type I, Gödel and Bertotti metrics. A special case is the Kerr metric, in two versions, one in Kerr-Schild coordinates and the other one in standard Kerr (Cartesian) coordinates. Our results are similar to those discussed above.

We have further studied the problem of introducing the Robertson-Walker ([16]) metric (2) as a generic case for the study of numerical cosmology. Here we use the scale factor of the universe $R(t)$ as a variable function through the code, and two initial parameters: the initial mass-energy density and the initial scale factor of the universe. Because this case has several specific problems and features we shall report on it in a future article in preparation. This is intended to be done in connection with the further development of the Exact thorn code, where we shall introduce a scalar field coupled with gravitation in order to study inflationary models.

\section{Acknowledgments}

D.N.V. was supported, for one of his visits to the AEI, by the DAAD through a NATO advanced research fellowship. He is also deeply indebted to Prof. E. Seidel for patience, continuous support and encouragement, making possible his introduction to numerical relativity and to the Cactus code.

\section{References}

[1] Misner C.W., Thorne K.S., Wheeler J.A. : Gravitation, Freeman, San Francisco, (1973)

[2] d'Inverno R.: Introducing Einsten's Relativity, Clarendon Press, Oxford, (1992)

[3] Arnowitt R., Deser S., Misner C.W. : Gravitation - an introduction to current research, ed. by L. Witten, John Wiley and Sons, New York, (1962)

[4] Hehl F.W., Puntigam R., Ruder H. (eds.) : Relativity and Scientific Computing, Springer Verlag, see the lectures of E. Seidel (p.25), P. Laguna (p. 88) and C. Bona (p. 69) , (1996) 
[5] Laguna P., The Grand Challenge in Computational Gravitation :Collision of BlackHoles, in Vulcanov D.N., Cotăescu I.I (eds.), - Gravity, Theoretical Physics and Computers, Proceedings of the VI-th conference in General Relativity and Gravitation, Bistriţa, Romania, 1996, Mirton Publ.House, Timişoara, (1997) (see also the references cited here)

[6] http://www.cactuscode.org and E. Seidel, Wai-Mo Suen, Journ. Comp. Appl. Math., 109, p. 493, (1999), B. Brügmann, Ann. Phys. (Leipzig), 9, No. 3-5, p. 227 (2000)

[7] M. Alcubierre : Integration of geodesics as a testbed for comparing exact and numerical spacetimes. Talk given at the 15th International Conference on General Relativity and Gravitation (GR15), IUCCA, Pune, India, December 16-21, 1997.

[8] C. Bona, J. Masso, E. Seidel. P. Walker, gr-qc/9804052, (1998)

[9] M. Alcubierre, et al., Phys. Rev. D60, 064017, (1999)

[10] M. Alcubierre, et al., Class. Quantum. Grav., 17, 2159, (2000)

[11] M. Alcubierre et al., Phys. Rev. D61, 041501, Phys. Rev. D62, 044034, 124011, Phys. Rev. D63, 104006, (2000)

[12] M. Alcubierre, et al., gr-qc/0012079, (2000)

[13] M. Alcubierre, et al., gr-qc/0104020, Report no. AEI-2001-021, (2001)

[14] T. W. Baumgarte and S. L. Shapiro, Phys. Rev. D 59, 024007, (1999)

[15] M. Shibata, T. Nakamura, Phys. Rev. D 52, 5428, (1995)

[16] J.N. Islam, An Introduction to Mathematical Cosmology, Cambridge University Press, Cambridge, (1992)

[17] S. Gotlober, et. al. : Early Evolution of the Universe and Formation Structure, Akad. Verlag, Berlin, (1990)

[18] S.D. Hern, Numerical Relativity and Inhomogeneous Cosmologies, PhD thesis, Cambridge, http::xxx.lanl.gov/gr-qc/0004036 and S.D. Hern, J.M. Stewart, Class.Quantum Grav, 15, 1581, (1998) 
[19] L. Pimentel, Int. Journ. of Theor. Physics, 32, No. 6, p. 979, (1993) (and the references cited here), 